\title{
Isolamento e perfil enzimático de cães e gatos com dermatofitose atendidos em hospitais veterinários do Recife, Pernambuco ${ }^{1}$
}

\author{
Michelly E.L.M. Torres², Polyanna N. Herculano², Maria L.F. Lima³, \\ Paola T. Soares ${ }^{3}$, Ana B.S. Siqueira ${ }^{4}$, Cristina M. Souza-Motta ${ }^{5}$, Ana L.F. Porto ${ }^{2,6}$ \\ e Cynthia O. Nascimento ${ }^{2 *}$
}

\begin{abstract}
Torres M.E.L.M., Herculano P.N., Lima M.L.F., Soares P.T., Siqueira A.B.S., Souza-Motta C.M., Porto A.L.F. \& Nascimento C.O. 2018. [Isolation and enzymatic profile of dogs and cats with dermatofitosis attended at veterinary hospitals in Recife, Pernambuco, Brazil.] Isolamento e perfil enzimático de cães e gatos com dermatofitose atendidos em hospitais veterinários do Recife, Pernambuco. Pesquisa Veterinária Brasileira 38(5):930-934. Laboratório de Tecnologia de Bioativos, Departamento de Morfologia e Fisiologia Animal, Universidade Federal Rural de Pernambuco, Rua Dom Manoel de Medeiros s/n, Dois Irmãos, Recife, PE 52171-900, Brazil. E-mail: cynthiaonbm@yahoo.com.br

Dermatophytes are fungi that can cause superficial infections of the skin, hair and nails in man and animals. The most frequent dermatophyte species isolated from dogs and cats are Microsporum gypseum, most notably Microsporum canis. The crucial role during the infection process is the production of extracellular enzymes essential for the invasion and establishment of the pathogen in the host tissue. The objective of this research was to isolate dermatophytes from dogs and cats and evaluate the enzymatic profile of the isolates obtained. Hair samples and epidermal scales were collected from dogs and cats in veterinary facilities in Recife-PE, and the isolates were identified based on macroscopic and microscopic characteristics. The qualitative analysis of the enzymes urease, protease, lipase, collagenase and phospholipase was evaluated from the isolated dermatophytes. During 10 months, a total of 106 animals, comprising of 99 dogs and seven cats with clinical signs, regardless of sex and race were evaluated. Only eight animals were confirmed with dermatophytosis, mostly dogs ( $\mathrm{n}=7)$, being six affected by $M$. canis and one by M. gypseum, the race most affected was Yorkshire $(\mathrm{n}=3)$. However, only one cat was confirmed with M. canis. No sex-related predisposition was observed regarding the occurrence of dermatophytosis in dogs and cats evaluated. Isolated dermatophytes showed similar profiles for the enzymes urease, lipase, protease, phospholipase and collagenase, important characteristic for pathogenic infections. The diagnosis of this zoonosis based on microbiological confirmation and a better understanding of the underlying mechanisms is of great importance for the treatment and prevention of fungal diseases in animals.
\end{abstract}

INDEX TERMS: Cats, dogs, Microsporum canis, Microsporum gypseum, dermatophytosis, enzymatic activity, clinics.

\footnotetext{
${ }^{1}$ Recebido em 28 de março de 2016.

Aceito em 25 de abril de 2017.

${ }^{2}$ Departamento de Morfologia e Fisiologia Animal, Universidade Federal Rural de Pernambuco (UFRPE), Rua Dom Manoel de Medeiros s/n, Dois Irmãos, Recife, PE 52171-900, Brasil. *Autor para correspondência: cynthiaonbm@yahoo.com.br

${ }^{3}$ Hospital Veterinário Harmonia, Estrada do Encanamento 585, Casa Forte, Recife, PE 52070-000.
}

\footnotetext{
${ }^{4}$ Departamento de Ciências Farmacêuticas, Universidade Federal de Pernambuco (UFPE), Av. Prof. Arthur de Sá s/n, Cidade Universitária, Recife, PE 50740-520.

${ }^{5}$ Departamento de Micologia, Universidade Federal de Pernambuco (UFPE), Av. Prof. Moraes Lins do Rego s/n, Cidade Universitária, Recife, PE 50670-420.

${ }^{6}$ Laboratório de Imunopatologia Keizo Asami (LIKA), Universidade Federal de Pernambuco (UFPE), Av. Prof. Moraes Lins do Rego s/n, Recife, PE 50670-901.
} 
RESUMO.- Os dermatófitos são fungos que podem causar infecções superficiais da pele, cabelo e unhas em humanos e animais. As espécies de dermatófitos mais frequentemente isoladas dos cães e gatos afetados por micoses são Microsporum gypseum e principalmente Microsporum canis. 0 papel crucial durante o processo de infecção é a produção de enzimas extracelulares essenciais para a invasão e estabelecimento do agente patogênico no tecido do hospedeiro. 0 objetivo deste trabalho foi isolar dermatófitos de cães e gatos e avaliar o perfil enzimático dos isolados obtidos. Amostras de pelos e escamas epidérmicas foram coletadas de cães e gatos em instalações veterinárias em Recife/PE, e os isolados foram identificados com base nas características macroscópicas e microscópicas. A análise qualitativa das enzimas urease, protease, lipase, colagenase e fosfolipase foi avaliada a partir dos dermatófitos isolados. Durante 10 meses, um total de 106 animais, que compreendeu de 99 cães e sete gatos com sinais clínicos, independentemente do sexo e raça foram avaliados. Apenas oito animais foram confirmados com dermatofitose, principalmente cães ( $\mathrm{n}=7$ ), sendo seis afetados por M. canis e um por M. gypseum, a raça mais afetada foi Yorkshire ( $\mathrm{n}=3$ ). No entanto, apenas um gato foi confirmado com M. canis. Não foi observada predisposição relacionada ao sexo quanto à ocorrência de dermatofitose nos cães e gatos avaliados. Os dermatófitos isolados apresentaram perfis semelhantes para as enzimas urease, lipase, protease, fosfolipase e colagenase, característica importante em infecções patogênicas. 0 diagnóstico clínico destas zoonoses com base na confirmação microbiológica e uma compreensão dos mecanismos subjacentes é de grande importância para o tratamento e prevenção de doenças fúngicas em animais.

TERMOS DE INDEXAÇÃO: Gatos, cães, Microsporum canis, M. gypseum, dermatofitose, atividade enzimática, clínica.

\section{INTRODUÇÃO}

Os dermatófitos são fungos que pertencem aos gêneros Trichophyton, Microsporum e Epidermophyton (Brasch 2010, Segal \& Frenkel 2015). São capazes de causar infecções na pele, no cabelo, pelos e unhas (Siqueira et al. 2009), denominadas de dermatofitoses, que são altamente contagiosas. Dermatofitose é "uma infecção zoonótica, transmissível, em condições naturais, dos animais vertebrados ao homem", por contato direto de lesões e fômites contaminados (Cabañes 2000, Madrid \& Mattei 2011). Os animais domésticos, especialmente cães e gatos, desempenham um papel importante na disseminação dos dermatófitos por serem uma fonte primária e direta de infecção (Beraldo et al. 2011, Sheinberg et al. 2017).

De acordo com seus habitats naturais, os dermatófitos podem ser classificados como antropofílicos, geofílicos e zoofílicos. Dermatófitos zoofílicos, como Microsporum canis e Trichophyton mentagrophytes e aqueles geofílicos como $M$. gypseum, são agentes etiológicos frequentemente isolados de animais domésticos com dermatofitoses (Ilhan et al. 2016, Roshanzamir et al. 2016).

As infecções produzidas por dermatófitos são geralmente superficiais, afetam fundamentalmente o estrato córneo da pele e anexos, assim como a superfície das mucosas (Diego 2011) e são caracterizadas principalmente por alopecias multifocais e descamações (Seker \& Dogan 2011).
A patogênese da dermatofitose começa pela aderência de estruturas reprodutivas dos dermatófitos, seguida pela formação de hifas que podem se espalhar pelo tecido do animal. Durante a penetração, os dermatófitos secretam uma variedade de enzimas virulentas, tais como protease, lipase, fosfolipase e colagenase, as quais possuem diferentes especificidades por substrato, sendo esses os nutrientes fundamentais para a sobrevivência dos dermatófitos e o estabelecimento da doença no hospedeiro (Brasch 2010, Sheinberg et al. 2017).

A secreção de uma ampla variedade de enzimas por dermatófitos é um dos fatores mais importantes durante o processo infeccioso, pois possibilitam que esses patógenos se tornem invasivos. Lipases, proteases e fosfolipases desempenham um papel importante na fase inicial de invasão dos dermatófitos no estrato córneo da pele no hospedeiro e podem ter relevância na patogênese de dermatofitoses animais (Peres et al. 2010, Cafarchia et al. 2012).

0 objetivo deste trabalho foi isolar dermatófitos de amostras clínicas de cães e gatos atendidos em hospitais veterinários da cidade de Recife/PE e avaliar o perfil enzimático dos dermatófitos obtidos.

\section{MATERIAL E MÉTODOS}

\section{Animais}

As amostras clínicas (pelos e escamas epidérmicas) foram coletadas de cães e gatos com sinais clínicos sugestivos de dermatofitose, para exame micológico em um hospital veterinário público e um privado da cidade de Recife/PE. Os animais foram avaliados independentemente do sexo e raça, no período de março/2011 a janeiro/2012.

\section{Isolamento e identificação dos dermatófitos}

O isolamento foi realizado através da coleta por escarificação das lesões com o auxílio de bisturis esterilizados e descartáveis, sendo as amostras clínicas acondicionadas em recipientes esterilizados. Para obtenção da cultura, as amostras foram semeadas em placas de Petri contendo meio Ágar Sabouraud Dextrose (ASD), acrescido de cloranfenicol 50mg/L. As mesmas foram incubadas em estufa microbiológica à temperatura de $30^{\circ} \mathrm{C}$ no período de 15 a 20 dias. As colônias fúngicas sugestivas de dermatófitos foram isoladas e mantidas em meio Batata-Dextrose-Ágar (BDA), para posterior identificação.

A identificação foi realizada de acordo com as características macroscópicas e microscópicas, segundo os critérios adotados por Lacaz et al. (2002). Os isolados foram crescidos em tubo de ensaio contendo meio ASD acrescido de extrato de levedura e preservados sob o método de óleo mineral (Sherf 1943).

\section{Perfil enzimático}

Para determinar o potencial enzimático para as enzimas protease, lipase, fosfolipase, colagenase e urease foram utilizados meios sólidos com os respectivos substratos. Fragmentos de colônias dos dermatófitos foram colocados no centro das placas de Petri com os meios específicos, e incubados a $30^{\circ} \mathrm{C}$ por até 20 dias, exceto para atividade de urease cuja avaliação foi realizada após 96 horas de incubação. 0 potencial enzimático para as enzimas protease, lipase e fosfolipase foi avaliado pelo cálculo da zona de atividade (ZA): (diâmetro da colônia $(\mathrm{mm}) /$ (diâmetro da colônia mais diâmetro do halo ( $\mathrm{mm}$ ), porém para as enzimas lipase e fosfolipase a zona de atividade foi denominada de zona de precipitação (ZP).

Detecção de protease. 0 meio de caseína, composto de leite desnatado $10 \%$ (p/v) e ágar bacteriológico $2 \%(\mathrm{p} / \mathrm{v}$ ) foi utilizado 
para detecção de protease, segundo metodologia proposta por Kantarcioglu \& Yücel (2002). A formação de zona de atividade ao redor da colônia indicou a produção desta enzima. 0 potencial de produção desta enzima foi avaliado através dos valores da ZA, sendo: $\mathrm{ZA}=0,9-1,0$ (potencial muito baixo $(+$ ); $\mathrm{ZA}=0,80-0,89$ (potencial baixo $(++)$; ZA=0,70-0,79 (potencial elevado $(+++$ ); $\mathrm{ZA}<0,69$ (potencial muito elevada $(++++)$.

Detecção de lipase. Para avaliação da enzima lipase foi utilizado o meio composto de peptona $1 \%(\mathrm{p} / \mathrm{v})$, cloreto de sódio $0,5 \%(\mathrm{p} / \mathrm{v})$, cloreto de cálcio 0,01\% (p/v), ágar 2\% (p/v) e de Tween 20 1\% (v/v). A formação de zona de precipitação (ZP) foi indicativo de potencial lipolítico, sendo negativo para valor $\mathrm{ZP}=1,0$ e positivo para valores $\mathrm{ZP}<1,0$ (Santos et al. 2001).

Detecção de fosfolipase. Esta enzima foi detectada utilizandose o meio contendo lecitina de soja $0,2 \%(\mathrm{p} / \mathrm{v})$, dextrose $4 \%(\mathrm{p} / \mathrm{v})$, peptona de carne $1 \%(\mathrm{p} / \mathrm{v})$, cloreto de sódio $0,03 \%(\mathrm{p} / \mathrm{v})$, cloreto de cálcio $0,05 \%(\mathrm{p} / \mathrm{v}$ ) e ágar bacteriológico $2 \%(\mathrm{p} / \mathrm{v}), \mathrm{pH} 7,0 \pm 0,2$ segundo metodologia de Price \& Cawson (1977). A formação de zona de precipitação (ZP) foi indicativo de produção de fosfolipase, sendo negativo para valor $\mathrm{ZP}=1,0$ e positivo para valores $\mathrm{ZP}<1,0$ (Santos et al. 2001).

Detecção de colagenase. 0 meio para colagenase foi gelatina $1 \%$ (p/v) e ágar 2\% (p/v). 0 crescimento em meio sólido contendo gelatina como fonte de carbono e nitrogênio foi indicativo da produção de colagenase pelos dermatófitos avaliados (Wawrzkiewicz et al. 1991).

Detecção de uréase. A enzima urease foi detectada em meio de Christensen, composto de peptona $0,1 \%(\mathrm{p} / \mathrm{v})$, dextrose $0,1 \%(\mathrm{p} / \mathrm{v})$, cloreto de sódio $0,5 \%(\mathrm{p} / \mathrm{v})$, fosfato dissódico $0,1 \%(\mathrm{p} / \mathrm{v})$, fosfato monopotássico $0,08 \%(\mathrm{p} / \mathrm{v})$, vermelho fenol 0,0012\% (p/v), solução de uréia $40 \%(\mathrm{p} / \mathrm{v})$ e ágar $1,5 \%(\mathrm{p} / \mathrm{v}), \mathrm{pH} 6.8 \pm 0.2$. 0 resultado foi considerado positivo quando a coloração do meio modificou de amarelo para róseo indicando que houve degradação da ureia e considerado negativo quando o meio permaneceu amarelo (Sidrim \& Moreira 1999, Sidrim \& Rocha 2004).

\section{RESULTADOS E DISCUSSÃO}

\section{Isolamento e identificação dos dermatófitos}

Um total de 106 animais foi avaliado em relação à presença de dermatofitose, dos quais 99 eram cães e sete eram gatos. 0 número menor de gatos pode ser justificado pelo fato de terem sido analisados apenas animais com sinais clínicos sugestivos para dermatofitose e os gatos serem considerados carreadores assintomáticos de artrósporos dermatofíticos (Farias et al. 2011).

Do total de animais (106) foram isolados oito dermatófitos, sete identificados como Microsporum canis e um M. gypseum. Estudos sobre isolamento de dermatófitos em cães e gatos com suspeita de dermatofitose têm sido reportados por diversos autores. Beraldo et al. (2011) realizaram o isolamento e a identificação de dermatófitos a partir de escamas de pele coletadas de 40 cães e 40 gatos, dos quais foram obtidos dermatófitos em 13 cães e 14 gatos, sendo M. canis a espécie prevalente entre os gatos e Trichophyton spp. entre os cães. Yamamura et al. (1997), avaliaram amostras clínicas de 103 animais (86 cães e 17 gatos) e do total obtiveram nove dermatófitos, com predominância para $M$. canis, dados semelhantes ao encontrado neste trabalho. Seker \& Dogan (2011) e Debnath et al. (2016) em seus estudos de isolamento de dermatófitos em cães e gatos também relataram $M$. canis como a espécie mais predominante nestes animais.
O Quadro 1 apresenta o número das espécies de dermatófitos isolados de acordo com o animal hospedeiro. Dentre os 99 cães, foram isolados sete dermatófitos, seis M. canis e um M. gypseum, enquanto que, dos sete gatos, apenas um foi confirmado com dermatofitose, sendo isolado um M. canis. Silva et al. (2011) avaliaram amostras de 41 cães e sete gatos, das quais isolaram seis dermatófitos em cães, destes, três foram identificados como M. canis, dois M. gypseum e um M. nanum, no entanto não foram obtidos dermatófitos em gatos.

Nesse trabalho, dos 99 cães, 48 eram machos e 51 eram fêmeas, em relação aos gatos, dois eram machos e cinco eram fêmeas. Dos sete dermatófitos isolados em cães, três foram obtidos de machos e quatro a partir de fêmeas. 0 dermatófito isolado de gato foi oriundo de uma fêmea. Não foi observada predisposição relacionada ao sexo quanto à ocorrência de dermatofitose nos cães e gatos avaliados, corroborando com os dados relatados por Palumbo et al. (2010), os quais, num estudo epidemiológico realizado para definir as características da ocorrência da dermatofitose na população canina e felina, atendida pelo serviço de Dermatologia Veterinária da FMVZ-UNESP campus de Botucatu, não observaram diferença estatística quanto ao sexo dos animais acometidos com dermatofitose.

Dentre os animais selecionados, 39 cães não apresentaram raça definida (SRD) e 60 tinham padrão racial: 15 Poodle, 12 Yokshire, sete Dachshund e os demais 26 de raças diversas (Fox Paulistinha (3), Shitzu (3), Labrador (3), Beagle (3), Border Collie (3), Pinscher (2), Pitbull (2), Pastor Alemão (2), Buldog (1), Golden (1), Pug (1), Chow Chow (1), American Staffordshire Terrier (1). Os sete gatos não possuíam definição racial, todos os animais, inclusive os cães, eram domiciliados.

Dos sete dermatófitos isolados de cães, três foram obtidos de cães da raça Yorkshire, um da raça Chow Chow, um da raça Pitbull e dois de cães sem raça definida. Neves et al. (2011) realizaram análise retrospectiva dos prontuários de atendimentos de cães e gatos com suspeita de dermatofitoses no Hospital Veterinário da Universidade Federal de Mato Grosso, sendo a dermatofitose diagnosticada em 279 dos 3906 de casos atendidos em três anos. Estes autores observaram maior número de casos positivos para dermatofitose em cães com raça definida, fato também notado nesta pesquisa.

A raça Yorkshire representou a maior parte dos casos de dermatofitose, corroborando com Balda et al. (2004) que descrevem esta raça como a mais acometida. Segundo Roshanzamir et al. (2016) a raça Yorkshire apresenta maior prevalência de dermatofitoses, principalmente causada por $M$. canis. Vários autores divergem entre si em relação às causas da predisposição desta raça. Sparkes et al. (1993) mencionam o tamanho do pelo como um fator importante, uma vez que pelos alongados protegem as estruturas fúngicas da dissecação, favorecendo a propagação das mesmas, devido às ótimas condições de temperatura e umidade. No entanto, essa predisposição se deve às diferenças nas defesas cutâneas como secreção sebácea/sudorípara e não devido ao seu pelo longo.

Quadro 1. Dermatófitos isolados de cães e gatos atendidos em Hospitais Veterinários do Recife/PE

\begin{tabular}{lccc}
\hline \multicolumn{1}{c}{ Dermatófito } & Total $(\mathrm{n}=106)$ & Cão $(\mathrm{n}=99)$ & Gato $(\mathrm{n}=7)$ \\
\hline Microsporum canis & 7 & 6 & 1 \\
Microsporum gypseum & 1 & 1 & 0 \\
Total & 8 & 7 & 1
\end{tabular}


A dermatofitose em cães e gatos é uma zoonose de grande importância em saúde pública devido ao contato do homem com estes animais. Em humanos, dados epidemiológicos indicam que a dermatofitose está entre as zoonoses mais comuns do mundo, sendo considerada a terceira doença de pele mais comum em crianças menores de 12 anos e a segunda em adultos (Neves et al. 2011). Por isso, a necessidade da realização de um diagnóstico preciso é relevante para o controle dessa micose nos animais domésticos e prevenção da contaminação em humanos (Galiza et al. 2014).

\section{Perfil enzimático dos dermatófitos}

O perfil enzimático dos dermatófitos é importante para a patogenicidade destes microrganismos, pois, após aderência de estruturas reprodutivas dos dermatófitos, ocorre a liberação de enzimas e substâncias tóxicas ou alergênicas, levando à ruptura da camada superficial da pele, proliferação no estrato córneo acompanhado de uma reação inflamatória do folículo piloso, epiderme e derme, causando queda de pelos, descamação, eritema e prurido. 0 processo inflamatório leva ao crescimento centrífugo do fungo, resultando no desenvolvimento de lesões circulares e alopecias (Madrid \& Mattei 2011).

O Quadro 2 mostra o perfil enzimático dos dermatófitos isolados de cães e gatos em relação as enzimas protease, lipase, fosfolipase, colagenase e urease. Todos os dermatófitos apresentaram atividade para enzimas testadas. De acordo com a literatura, os dermatófitos produzem uma variedade de enzimas de virulência, as quais estão envolvidas na patogênese dos dermatófitos, sendo a protease uma das mais importantes nesse processo, facilitando a invasão e penetração no estrato córneo do hospedeiro (Chinnapun 2015). De acordo com Cafarchia et al. (2012) a produção enzimática pode ser relacionada com a composição da pele, a qual influencia o papel patogênico de cada dermatófito em relação ao hospedeiro específico.

De maneira geral, as espécies $M$. canis e $M$. gypseum apresentaram perfis enzimáticos semelhantes, com resultado positivo para as enzimas avaliadas. Da mesma forma, Bruguera et al. (1997) observaram resultados semelhantes para o perfil enzimático de dermatófitos procedentes de dermatofitoses em humanos e animais. Entretanto, segundo Muhsin \& Salih (2001) o perfil enzimático pode variar de acordo com a espécie de dermatófito.

Quadro 2. Perfil enzimático dos dermatófitos isolados de cães e gatos atendidos em Hospitais Veterinários do Recife/PE

\begin{tabular}{|c|c|c|c|c|c|}
\hline \multirow[b]{2}{*}{ Dermatófito } & \multicolumn{4}{|c|}{ Enzimas } & \multirow[b]{2}{*}{ Urease } \\
\hline & $\begin{array}{c}\text { Protease } \\
(\mathrm{ZA})\end{array}$ & $\begin{array}{c}\text { Lipase } \\
\text { (ZP) }\end{array}$ & $\begin{array}{c}\text { Fosfolipase } \\
\text { (ZP) }\end{array}$ & Colagenase & \\
\hline $\begin{array}{l}\text { Microsporum } \\
\text { canis (2) }\end{array}$ & $++(0,84)$ & $+(0,76)$ & $+(0,82)$ & + & + \\
\hline M. canis (3) & $++(0,83)$ & $+(0,83)$ & $+(0,90)$ & + & + \\
\hline M. canis (14) & $+(1,00)$ & $+(0,76)$ & $+(0,90)$ & + & + \\
\hline M. canis (15) & $+(0,93)$ & $+(0,64)$ & $+(0,78)$ & + & + \\
\hline M. canis (16) & $+(0,93)$ & $+(0,79)$ & $+(0,77)$ & + & + \\
\hline M. canis (17) & $++(0,89)$ & $+(0,75)$ & $+(0,81)$ & + & + \\
\hline M. canis (18) & $+(1,00)$ & $+(0,63)$ & $+(0,94)$ & + & + \\
\hline M. gypseum (31) & $+(0,95)$ & $+(0,67)$ & $+(0,90)$ & + & + \\
\hline
\end{tabular}

$\overline{\mathrm{ZA}}=$ zona de atividade, $\mathrm{ZP}=$ zona de precipitação.
Foi observada diferença para atividades de proteases nas amostras de $M$. canis, analisadas neste estudo, para protease, onde três isolados apresentaram atividade baixa $(++)$ e os demais demonstraram atividade muito baixa $(+)$, levando-se em consideração o tamanho da zona de atividade. Maia et al. (2001) também relataram diferença na atividade de protease em amostras de $M$. canis, demonstrando que variações quanto à atividade enzimática podem ser observadas entre isolados de uma mesma espécie.

Em relação à avaliação da atividade da enzima urease, todos os isolados apresentaram resultado positivo. Resultados diferentes foram obtidos por Brilhante et al. (2005), os quais observaram que somente $80 \%$ dos dermatófitos $M$. canis produziram urease. Estudos tem sugerido que esta enzima apresenta papel importante durante infecções microbianas.

A atividade das enzimas fosfolipase e lipase produzidas pelos dermatófitos facilita o processo de infecção por romperem inicialmente a camada superficial lipídica da pele, favorecendo a colonização do estrato córneo da pele (Hellgren \& Vincent 1980, Elavarashi et al. 2017). A função da colagenase é degradar o colágeno presente na pele do hospedeiro, colaborando com a patogênese destes fungos. Segundo Viani et al. (2007), haveria uma relação entre os níveis de produção de colagenase e a presença ou não de dermatofitoses, como também foi estabelecida por Viani et al. (2001) a relação direta entre protease e patogenicidade de dermatófitos. Porém, poucos estudos são relatados para hidrolases, como lipase, entre outras enzimas produzidas por estes fungos (Vermout et al. 2008).

\section{CONCLUSÕES}

Os resultados demonstraram que Microsporum canis foi a espécie mais isolada de cães e gatos, sendo Yorkshire a raça mais acometida entre os cães. Não foi observada predisposição relacionada ao sexo dos animais, quanto à ocorrência de dermatofitose.

0 presente trabalho demonstra a importância do diagnóstico e confirmação da espécie fúngica em casos suspeitos de dermatofitoses para o acompanhamento e tratamento adequado desses pacientes, assim como o estabelecimento das medidas profiláticas adequadas no intuito de evitar a infecção e/ou reinfecção da doença. Todos os dermatófitos apresentaram perfil enzimático semelhante, demonstrando o potencial patogênico dos isolados avaliados.

Agradecimentos.- Os autores agradecem o apoio financeiro e bolsa concedida pela CAPES, FACEPE (BCT 0284-5.05/11) e CNPq.

\section{REFERÊNCIAS}

Balda A.C., Larsson C.E., Otsuka M. \& Gambale W. 2004. Estudo retrospectivo de casuística das dermatofitoses em cães e gatos atendidos no Serviço de Dermatologia da Faculdade de Medicina Veterinária e Zootecnia da Universidade de São Paulo. Acta Scient. Vet. 32(2):133-140.

Beraldo R.M., Gasparoto A.K., Siqueira A.M. \& Dias A.L.T. 2011. Dermatophytes in household cats and dogs. Revta Bras. Ciênc. Vet. 18(2/3):85-91.

Brasch J. 2010. Pathogenesis of tinea. J Dtsch Dermatol Ges 8(10):780-786. PMid:20678152.

Brilhante R.S., Cordeiro R.A., Medrano D.J., Monteiro A.J., Sidrim J.J. \& Rocha M.F. 2005. Antifungal susceptibility and genotypical pattern of Microsporum canis strains. Can. J. Microbiol. 51(6):507-510. http://dx.doi.org/10.1139/ w05-024. PMid:16121230. 
Bruguera M.T., Abarca M.L., Bragulat M.R. \& Cabañes F.J. 1997. Determinación de actividades enzimaticas en cepas de Microsporum canis y Microsporum gypseum. Revta Iberoam. Micol. 14:119-124.

Cabañes F.J. 2000. Dermatophytes in domestic animals. Revta Iberoam. Micol. 17:104-108.

Cafarchia C., Figueredo L.A., Coccioli C., Camarda A. \& Otranto D. 2012. Enzymatic activity of Microsporum canis and Trichophyton mentagrophytes from breeding rabbits with and without skin lesions. Mycoses 55(1):45-49. http://dx.doi.org/10.1111/j.1439-0507.2010.01997.x. PMid:22175244.

Chinnapun D. 2015. Virulence factors involved in pathogenicity of dermatophytes. Walailak J. Sci. Tech. 12:573-580.

Debnath C., Mitra T., Kumar A. \& Samanta I. 2016. Detection of dermatophytes in healthy companion dogs and cats in eastern India. Iran J. Vet. Res. 17(1):20-24. PMid:27656224.

Diego A.M. 2011. Aspectos clínicos, diagnósticos y terapéuticos de las dermatofitosis. Enferm. Infecc. Microbiol. Clin. 29(3):33-39. http://dx.doi. org/10.1016/S0213-005X(11)70025-8.

Elavarashi E., Kindo A.J. \& Rangarajan S. 2017. Enzymatic and non-enzymatic virulence activities of dermatophytes on solid media. J. Clin. Diagn. Res. 11(2):DC23-DC25. PMid:28384862.

Farias M.R., Condas L.A.Z., Ramalho F., Bier D., Muro M.D. \& Pimpão C.T. 2011. Avaliação do estado de carreador assintomático de fungos dermatofíticos em felinos (Felis catus Linnaeus, 1793) destinados à doação em centros de controle de zoonoses e sociedades protetoras de animais. Vet. Zootec. 18(2):306-312.

Galiza G.J.N., Silva T.M., Caprioli R.A., Barros C.S.L., Irigoyen L.F., Fighera R.A., Lovato M. \& Kommers G.D. 2014. Ocorrência de micoses e pitiose em animais domésticos: 230 casos. Pesq. Vet. Bras. 34(3):224-232. http:// dx.doi.org/10.1590/S0100-736X2014000300005.

Hellgren L. \& Vincent L. 1980. Lipolytic activity of some dermatophytes. J. Med. Microbiol. 13(1):155-157. http://dx.doi.org/10.1099/0022261513-1-155. PMid:7359572.

Ilhan Z., Karaca M., Ekin I.H., Solmaz H., Akkan H.A. \& Tutuncu M. 2016. Detection of seasonal asymptomatic dermatophytes in Van cats. Braz. J. Microbiol. 47(1):225-230. http://dx.doi.org/10.1016/j.bjm.2015.11.027. PMid:26887249.

Kantarcioglu A.S. \& Yücel A. 2002. Phospholipase and protease activities in clinical Candida isolates with reference to the sources of strains. Mycoses 45(5/6):160-165. http://dx.doi.org/10.1046/j.1439-0507.2002.00727.x. PMid:12100532.

Lacaz C.S., Porto E., Martins J.E.C., Heins-Vaccari E.M. \& De Melo N.T. 2002. Tratado de Micologia Médica. 9aa ed. Sarvier, Rio de Janeiro. 1104p.

Madrid I.M. \& Mattei A.S. 2011. Dermatofitose, p.37-47. In: Ibid. (Eds), Manual de Zoonoses. Vol.II. CRMVRS, CRMVSC, CRMVPR, Rio Grande do Sul.

Maia M.L.S., Santos J.I., Viani F.C., Larsson C.E., Paula C.R. \& Gambale W. 2001. Phenotypic characterization of Microsporum canis isolates from cats and dogs. Mycoses 44(11/12):480-486. http://dx.doi.org/10.1046/j.14390507.2001.00702.x. PMid:11820261.

Muhsin T.M. \& Salih T.H. 2001. Exocellular enzyme activity of dermatophytes and other fungi solated from ruminants in Southern Iraq. Mycopathologia 150(2):49-52. http://dx.doi.org/10.1023/A:1010854322655. PMid:11407490.

Neves R. de C., Cruz F.A.C.S., Lima S.R., Torres M.M., Dutra V. \& Sousa V.R.F. 2011. Retrospectiva das dermatofitoses em cães e gatos atendidos no Hospital Veterinário da Universidade Federal de Mato Grosso, nos anos de 2006 a 2008. Ciência Rural 41(8):1405-1410. http://dx.doi.org/10.1590/ S0103-84782011000800017.

Palumbo M.I.P., Machado L.H. de A., Paes A.C., Mangia S.H. \& Motta R.G. 2010. Estudo epidemiológico das dermatofitoses em cães e gatos atendidos no serviço de dermatologia da Faculdade de Medicina Veterinária e Zootecnia da UNESP/Botucatu. SSemina, Ciênc. Agrárias 31(2):459-468.
Peres N.T.A., Maranhão F.C.A., Rossi A. \& Martinez-Rossi N.M. 2010. Dermatófitos: interação patógeno-hospedeiro e resistência a antifúngicos. Anais Bras. Dermatol. 85(5):657-667. http://dx.doi.org/10.1590/S036505962010000500009.

Price M.F. \& Cawson R.A. 1977. Phospholipase activity in Candida albicans. Sabouraudia 15(2):179-185. http://dx.doi.org/10.1080/00362177785190271. PMid:333624.

Roshanzamir H., Naserli S., Ziaie B. \& Fakour M. 2016. Incidence of dermatophytes isolated from dogs and cats in the city of Baku, Azerbaijan. Comp. Clin. Pathol. 25(2):327-329. http://dx.doi.org/10.1007/s00580-015-2185-x.

Santos J.I., Vicente E.J., Paula C.R. \& Gambale W. 2001. Phenotypic characterization of Trichophyton rubrum isolates from two geographic locations in Brazil. Eur. J. Epidemiol. 17(8):729-735. http://dx.doi.org/10.1023/A:1015675728486. PMid:12086090.

Segal E. \& Frenkel M. 2015. Dermatophyte infections in environmental contexts. Res. Microbiol. 166(7):564-569. http://dx.doi.org/10.1016/j. resmic.2014.12.007. PMid:25634072.

Seker E. \& Dogan N. 2011. Isolation of dermatophytes from dogs and cats with suspected dermatophytosis in Western Turkey. Prev. Vet. Med. 98(1):46-51. http://dx.doi.org/10.1016/j.prevetmed.2010.11.003. PMid:21126787.

Sheinberg G., Romero C., Heredia R., Casas D. \& Galicia E. 2017. Dermatophytes from a zoonotic point of view. Int. J. Curr. Adv. Res. 6(1):1856-1861.

Sherf A.F. 1943. A method for maintaining Phytomonas sepedonica in culture for long periods without transfer. Phytopathology 33:330-332.

Sidrim J.J.C. \& Moreira J.L.B. 1999. Fundamentos Clínicos e Laboratoriais da Micologia Médica. Guanabara-Koogan, Rio de Janeiro. 287p.

Sidrim J.J.C. \& Rocha M.F.G. 2004. Micologia Médica à Luz de Autores Conteporâneos. Guanabara-Koogan, Rio de Janeiro. 408p.

Silva V.F., Drescher G. \& Mattiello S.P. 2011. Agentes fúngicos da dermatofitose em cães e gatos do município de Xanxerê, Santa Catarina. Semina, Ciênc. Agrárias 32(3):1095-1100.

Siqueira A.B.S., Gomes B.S., Cambuim I., Maia R., Abreu S., Souza-Motta C.M., de Queiroz L.A. \& Porto A.L. 2009. Trichophyton species susceptibility to green and red propolis from Brazil. Lett. Appl. Microbiol. 48(1):90-96. http://dx.doi.org/10.1111/j.1472-765X.2008.02494.x. PMid:19018958.

Sparkes A.H., Gruffydd-Jones T.J., Shaw S.E., Wright A.I. \& Stokes C.R. 1993. Epidemiological and diagnostic features of canine and feline dermatophytosis in the United Kingdom from 1956 to 1991. Vet. Rec. 133(3):57-61. http:// dx.doi.org/10.1136/vr.133.3.57. PMid:8212483.

Vermout S., Tabart J., Baldo A., Mathy A., Losson B. \& Mignon B. 2008. Pathogenesis of dermatophytosis. Mycopathologia 166(5/6):267-275. http://dx.doi.org/10.1007/s11046-008-9104-5. PMid:18478361.

Viani F.C., Santos J.I., Paula C.R., Larson C.E. \& Gambale W. 2001. Production of extracellular enzymes by Microsporum canis and their role in its virulence. Med. Mycol. 39(5):463-468. http://dx.doi.org/10.1080/mmy.39.5.463.468. PMid:12054058.

Viani F.C., Viani P.R.C., Rivera I.N.G., Silva E.G., Paula C.R. \& Gambale W. 2007. Extracellular proteolytic activity and molecular analysis of Microsporum canis strains isolated from symptomatic and asymptomatic cats. Revta Iberoam. Micol. 24(1):19-23. http://dx.doi.org/10.1016/S1130-1406(07)70004-9. PMid:17592886.

Wawrzkiewicz K., Wolski T. \& Lobarzewski J. 1991. Sreening the keratinolytic activity of dermatophytes "in vitro". Mycopathologia 114(1):1-8. http:// dx.doi.org/10.1007/BF00436684. PMid:1713301.

Yamamura A.A.M., Pereira E.P., Shimada M.K., Fugiwara C.Y., Danhone A.S. \& Chami D. 1997. Ocorrência de dermatofitose em cães e gatos atendidos pelo Hospital Veterinário da Universidade Estadual de Londrina. Semina, Ciênc. Agrárias 18:41-44. 